\title{
Comparison of Time and Frequency Domain Single and Double Differential Schemes for OFDM UWB Systems
}

\author{
Samia Islam, Naofal Al-Dhahir, Hlaing Minn, Sili Lu \\ Department of Electrical Engineering, University of Texas at Dallas \\ Email: \{sri031000, aldhahir, hlaing.minn, sx1059000\}@utdallas.edu
}

\begin{abstract}
Ultra-wideband (UWB) systems possess several distinct features such as very high data rate, large multipath or frequency diversity and improved ranging precision while requiring low-power low-complexity transceivers. Among the different modulation schemes, differential PSK is an attractive option because it does not require channel estimation, and hence reduces implementation complexity. This paper investigates an OFDM UWB receiver based on both time and frequency-domain differential PSK modulation and compares the performances of single and double differential schemes in the presence of frequency offset.
\end{abstract}

\section{INTRODUCTION}

Ultra-wideband (UWB) systems possess several distinct features such as very high data rate, large multipath or frequency diversity and improved ranging precision, while requiring low power, low complexity transceivers. Applications of UWB include wireless personal area networks (WPANs), sensor networks, imaging systems, vehicular radar systems, etc [1]. A UWB receiver can be based on either a multi-carrier system where the bandwidth is divided into sub-channels, or a single-carrier system based on spread spectrum code division multiple access (SSCDMA) using the same total bandwidth. Orthogonal frequency division multiplexing (OFDM) receivers are most efficient at capturing multipath diversity, when the channel is highly dispersive. Additionally, OFDM systems have high spectral efficiency, inherent resilience to narrow-band radio frequency interference (RFI), and spectral flexibility [2]. Different modulation and demodulation techniques have been studied for the UWB system. A differential phase shift keying (PSK) system is an attractive noncoherent detection option because it eliminates the need for channel estimation, thus greatly reducing implementation complexity [3].

Differential modulation in an OFDM system can be implemented either in the time domain or the frequency domain, depending on the channel and the presence of frequency offset. If frequency offset is present, a firstorder differential (or single differential) scheme can yield poor performance. In that case, a second-order differential

The work of N. Al-Dhahir was supported by the Texas Advanced Technology Program (ATP) under Contract 009741-0023-2003 and by the National Science Foundation under Contracts CCF 04-30654 and DMS 05-28010 and by a gift from TI. (or double differential) scheme might be more robust to frequency offset. Single differential detection for UWB has been studied in [4]- [5], while a double-differential scheme for frequency-selective channels was analyzed in [9]. In this paper, we will provide a performance comparison of time and frequency-domain single and double differential schemes for OFDM-based UWB receivers.

This paper is organized as follows. Section II describes the UWB channel and OFDM system models. In Sections III and IV, single differential scheme for an OFDM system and double differential scheme are respectively described. Section V presents the simulation setup and results, and the paper is concluded in Section VI.

\section{MOdEL AND Assumptions}

The impulse response of a UWB multipath model from [2] is described as follows

$$
h(t)=S \sum_{l} \sum_{k} \alpha_{k, l} \delta\left(t-T_{l}-\tau_{k, l}\right)
$$

where $\alpha_{k, l}$ are the channel gain coefficients, $T_{l}$ is the delay of the $l$ th cluster, and $\tau_{k, l}$ is the delay of the $k$ th multipath component in the $l$ th cluster relative to the cluster arrival time. By definition, $\tau_{0, l}$ and $T_{0}$ are zeros. The multipath gain coefficients are calculated as follows

$$
\alpha_{k, l}=p_{k, l} \xi_{l} \beta_{k, l}
$$

where $\xi_{l}$ reflects the fading associated with the $l$ th cluster, $\beta_{k, l}$ reflects the fading associated with the $k$ th ray of the $l$ th cluster, and $p_{k, l}$ is an equiprobable +1 or -1 to account for signal inversion due to reflections.

The distribution of the fading is given below

$$
20 \log _{10}\left(\xi_{l} \beta_{k, l}\right) \sim \operatorname{Normal}\left(\mu_{k, l}, \sigma_{1}^{2}+\sigma_{2}^{2}\right)
$$

where the mean of the above distribution is defined as

$$
\mu_{k, l}=\frac{10 \ln \left(\Omega_{0}\right)-10 \frac{T_{l}}{\Gamma}-10 \frac{\tau_{k, l}}{\gamma}}{\ln (10)}-\frac{\left(\sigma_{1}^{2}+\sigma_{2}^{2}\right) \ln (10)}{20}
$$

$S$ represents the log-normal shadowing and is characterized by the following probability distribution

$$
20 \log _{10} S \sim \operatorname{Normal}\left(0, \sigma_{x}^{2}\right)
$$

The time-domain channel matrix $\mathbf{H}$ in an OFDM system is a Toeplitz matrix. If the cyclic prefix is greater 
than or equal to the maximum channel delay spread $\nu$, the equivalent channel matrix $\tilde{\mathbf{H}}$ is circulant and can be decomposed as follows

$$
\tilde{\mathbf{H}}=\mathbf{F}^{\prime} \mathbf{\Lambda} \mathbf{F}
$$

where $\mathbf{F}$ and $\mathbf{F}^{\prime}$ are the $N$-point unitary discrete Fourier transform (DFT) and inverse DFT (IDFT) matrices, respectively, and $\boldsymbol{\Lambda}$ is a diagonal matrix where the diagonal elements are equal to the $N$-point DFT coefficients of the channel. Therefore, if the IDFT of the transmitted OFDM symbol is $\mathbf{x}$ and the additive white Gaussian noise (AWGN) vector is $\mathbf{z}$, the received TD OFDM symbol $\mathbf{y}$ can be written as follows

$$
\mathbf{y}=\tilde{\mathbf{H}} \mathbf{x}+\mathbf{z}
$$

If $\mathbf{Y}$ is the DFT of $\mathbf{y}$, then

$$
\mathbf{Y}=\mathbf{\Lambda} \mathbf{X}+\mathbf{Z}
$$

where $\mathbf{X}$ and $\mathbf{Z}$ are the DFT of $\mathbf{x}$ and $\mathbf{z}$, respectively. The channel has been perfectly diagonalized. However, this will not be the case in the presence of frequency offset. Carrier frequency offset is the difference between carrier frequencies of the transmitter and the receiver. It causes inter-carrier interference (ICI) and distorts the signal, therefore reducing the signal-to-noise ratio (SNR) [6]. Frequency offset effects can be modeled by the diagonal matrix $\mathbf{W}$, where the diagonal elements are $\left\{1, e^{j 2 \pi f_{0} / N}, \ldots, e^{j 2 \pi f_{0}(N+\nu-1) / N}\right\}, f_{0}$ being the normalized (by the subcarrier spacing) carrier frequency offset. The equivalent channel matrix in the time domain is now WH. Since this is not a Toeplitz matrix anymore, the equivalent channel matrix $\tilde{\mathbf{H}}$ is no longer circulant.

\section{Differential Modulation FOR OFDM SYSTEM}

If the differentially-modulated symbols are placed on adjacent OFDM symbols along the same sub-carrier, then the system is called time-domain differential modulation (TDDM). On the other hand, if the differentiallymodulated symbols are placed on adjacent sub-carriers within the same OFDM symbol, the system is called frequency-domain differential modulation (FDDM) [8]. An important assumption made in differential modulation is that the channel remains constant over at least two consecutive transmissions.

\section{A. Time-Domain Differential Modulation}

The relationship between the transmitted phase and the input data phase for a single differential system is

$$
\theta_{k}^{l}=\theta_{k-1}^{l}+\phi_{k}^{l}
$$

where $\phi_{k}$ is the input data phase and $\theta_{k}$ is the transmitted data phase. If $\mathbf{U}$ is the input OFDM symbol, the relationship between $\mathbf{X}$ and $\mathbf{U}$ becomes

$$
X_{k}^{l}=X_{k-1}^{l} U_{k}^{l}
$$

where $k$ denotes the OFDM symbol index and $l$ denotes the sub-carrier index. The current received symbol $\mathbf{Y}_{k}$ in the frequency domain is given as follows

$$
\mathbf{Y}_{k}=\boldsymbol{\Lambda}_{k} \mathbf{X}_{k}+\mathbf{Z}_{k}
$$

and the previous received symbol $\mathbf{Y}_{k-1}$ is

$$
\mathbf{Y}_{k-1}=\boldsymbol{\Lambda}_{k} \mathbf{X}_{k-1}+\mathbf{Z}_{k-1}
$$

where $\boldsymbol{\Lambda}_{k}$ and $\boldsymbol{\Lambda}_{k-1}$ are assumed to be equal. Because the UWB channel is slowly time varying, this is a reasonable assumption to make. If (10) and (12) are substituted in (11), the following equation is obtained

$$
Y_{k}^{l}=\left(Y_{k-1}^{l}-Z_{k-1}^{l}\right) U_{k}^{l}+Z_{k}^{l}
$$

The current information symbol $\mathbf{U}_{k}$ can be found based on the current and the previous received OFDM symbols without channel knowledge. The decision metric is

$$
\hat{U}_{k}^{l}=Y_{k-1}^{l *} Y_{k}^{l}
$$

\section{B. Frequency-Domain Differential Modulation}

The relationship between the information symbol and the transmitted symbol is

$$
X_{k}^{l}=X_{k}^{l-1} U_{k}^{l}
$$

The channel is assumed to remain constant over at least two sub-carriers of an OFDM symbol. The following equation is derived the same way as (13)

$$
Y_{k}^{l}=\left(Y_{k}^{l-1}-Z_{k}^{l-1}\right) U_{k}^{l}+Z_{k}^{l}
$$

The decision metric depends on the current and the previous received sub-carriers, and is given by

$$
\hat{U}_{k}^{l}=Y_{k}^{l-1 *} Y_{k}^{l}
$$

\section{Differential Modulation in the Presence OF FREQUENCY OFFSET}

Double differential quadrature PSK (D-DQPSK) is a modification of single DQPSK (S-DQPSK) by implementing a second-order system. If the frequency offset is constant, D-DQPSK eliminates the effects of frequency offset, and therefore, improves the performance of the system [9]. Block diagrams of a classical double differential transmitter and receiver from [10] are given in Figures 1 and 2, respectively. Each of the two single

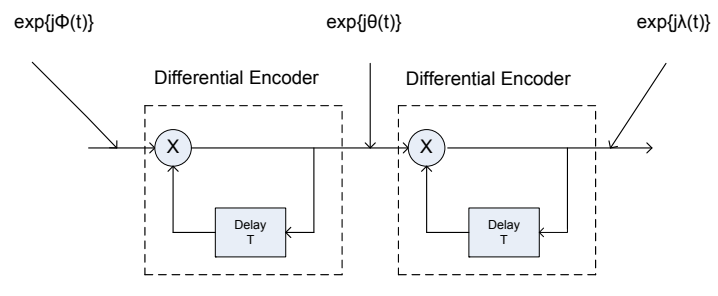

Fig. 1. Transmitter for double differential system

differential encoders and decoders can be applied in the time or frequency domain, and they work as described in Section III. From (9), the input to the second differential 
encoder is $\theta_{m}$, where $m$ is either the sub-carrier or the OFDM symbol index, depending on whether the differential modulation is being done in the time domain or the frequency domain. The second differential encoder output is

$$
\lambda_{m}=\lambda_{m-1}+\theta_{m}
$$

If $\mathbf{U}$ is the information, and $\mathbf{X}$ is to be transmitted, the relationship between them from [11] is

$$
\begin{aligned}
& \mathbf{V}_{m}=\mathbf{V}_{m-1} \odot \mathbf{U}_{m} \\
& \mathbf{X}_{m}=\mathbf{X}_{m-1} \odot \mathbf{V}_{m}
\end{aligned}
$$

where $\mathbf{V}$ is the output of the first differential encoder and $\odot$ is the Hadamard (element-wise) product. On the decoder side, the decision metric from (14) is passed through another differential decoder, before making a final decision as follows

$$
\begin{aligned}
& M_{m}=Y_{m-1}^{*} Y_{m} \\
& P_{m}=P_{m-1}^{*} M_{m}
\end{aligned}
$$

The final decision metric is then $P_{m}$. From [9], the noise output of the differential demodulator is given by $n(t)-$ $2 n(t-T)+n(t-2 T)$ where $T$ is the symbol period. An enhanced double differential system was implemented in [10] by choosing a delay of $2 T$ rather than $T$ in the first stage of the encoder and the second stage of the decoder. The classical and the enhanced systems will be referred to as $T / T$ and $T / 2 T$, respectively.

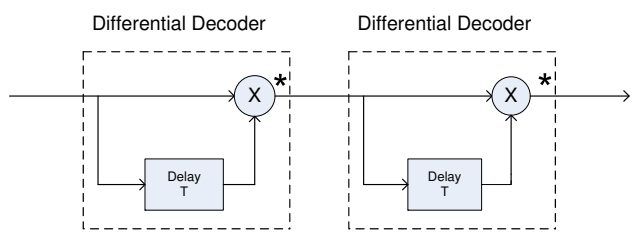

Fig. 2. Receiver for double differential system

The differential encoder output for the $T / 2 T$ system is

$$
\begin{aligned}
& \mathbf{V}_{m}=\mathbf{V}_{m-2} \odot \mathbf{U}_{m} \\
& \mathbf{X}_{m}=\mathbf{X}_{m-1} \odot \mathbf{V}_{m}
\end{aligned}
$$

and the decision metric becomes

$$
\begin{gathered}
M_{m}=Y_{m-1}^{*} Y_{m} \\
\hat{U_{m}}=U_{m-2}^{*} M_{m}
\end{gathered}
$$

The noise output in this scheme is given by $n(t)-n(t-$ $T)-n(t-2 T)+n(t-3 T)$. Because no noise terms are repeated, their effect is now reduced [9].

Note that the frequency-offset-induced matrix in the equivalent channel is $e^{j 2 \pi f_{0} m(N+v) / N} \mathbf{W}$ for the $m$ th OFDM symbol. This phase offset will introduce coherence loss to the TD single differential scheme and the coherent scheme. By applying the TD second differential operation, the above phase offset effect can be removed.

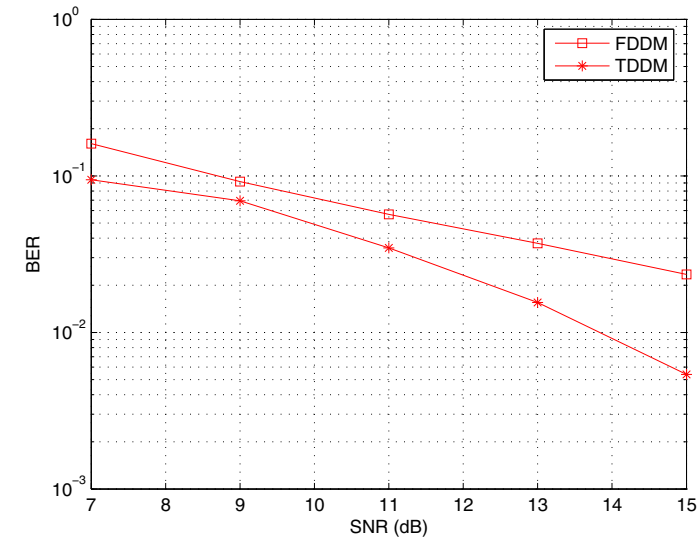

Fig. 3. BER vs. SNR for time and frequency-domain single differential scheme without frequency offset

\section{Simulation Setup and Results}

We have assumed that the transmission occurs in bursts, and consists arbitrarily of 13 OFDM symbols, over which the channel remains constant. The information bits were convolutionally encoded with a constraint length of 3 and a generator polynomial of [7,5]. The encoded bits are passed through a block interleaver of depth 16. QPSK modulation and UWB channel model (CM-1) were used for all simulations (see [2]). An equivalent low-pass channel was generated and used in the simulations. The number of sub-carriers (DFT size) was 128.

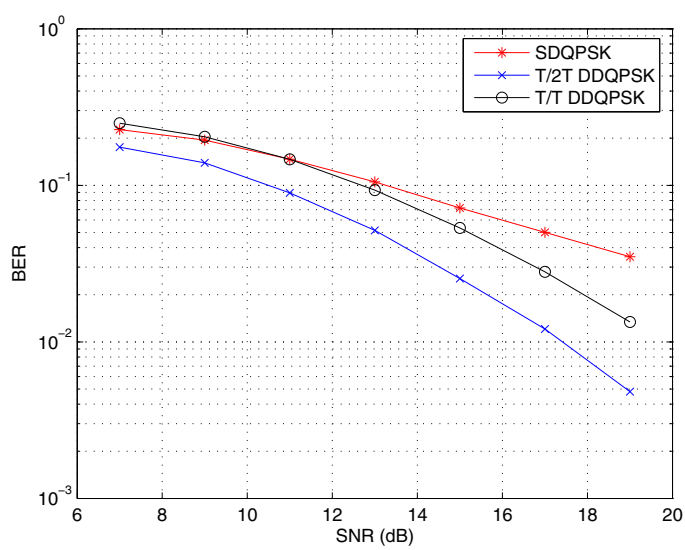

Fig. 4. BER vs. SNR for time-domain single and double differential scheme at a normalized frequency offset of 0.07

Fig. 3 shows the bit error rate (BER) comparison for time and frequency-domain single differential schemes in the absence of frequency offset. The TD differential scheme performs much better than the FD differential scheme. This is due to the time-invariant highlyfrequency-selective nature of the UWB channel. The FD single differential scheme suffers from the coherence loss between adjacent subcarrier channel gains while the TD scheme does not suffer from such a problem.

Fig. 4 presents the BER performances of TD single and double differential schemes in the presence of normalized frequency offset $f_{0}=0.07$. The TD double differential 
scheme outperforms the TD single differential scheme since the frequency-offset-induced coherence loss (phase offset) which affects the single differential scheme is cancelled out in the double differential schemes. The T/2T double differential scheme achieves about $2 \mathrm{~dB}$ SNR advantage over the $\mathrm{T} / \mathrm{T}$ double differential scheme which can be attributed to the larger noise variance (due to repeated noise term) in the $\mathrm{T} / \mathrm{T}$ scheme as mentioned in Section IV.

In Fig. 5, we present the BER performance of FD single and double differential schemes in the presence of a frequency offset $f_{0}=0.07$. The FD single differential scheme significantly outperforms the FD double differential scheme. This can be explained as follows. The frequency offset introduces ICI whose coefficients vary slowly except over adjacent subcarriers. Consequently, the first differential output at the receiver contains differential of correlated ICI terms. The second differential operation amplifies the effect of the ICI terms. Furthermore, the differential operation on the adjacent subcarrier channel gains will not yield a constant value and hence a second differential operation will amplify (rather than suppress) the coherence loss due to the channel frequency selectivity. The second differential operation also amplifies the noise effect. These effects explain the advantage of the FD single differential scheme over the FD double differential scheme.

The BER comparisons of coherent QPSK, FD single differential scheme and TD double differential (T/2T) scheme in the presence of frequency offset $f_{0}=0.07$ are presented in Fig. 6. At high SNR, the differential schemes outperform the coherent QPSK scheme. This can be explained as follows. The BER of coherent QPSK is caused by noise, frequency-offset-induced phase offsets and ICI, while the BERs of the differential schemes are affected by enhanced noise (larger for double differential), ICI and coherence loss due to frequency selectivity (for FD differential scheme). At moderate and low SNR, the noise effect dominates and hence the coherent scheme outperforms the differential schemes. At high SNR, the enhanced noise effect in the differential schemes becomes insignificant while the coherent scheme is affected by the frequency-offset-induced phase offsets which are canceled out in the differential schemes. Hence, the differential schemes gain advantage over the coherent scheme at high SNR.

Fig. 7 presents the BER comparisons of TD T/2T double and single differential, and FD single differential schemes at a normalized frequency offset of 0.03 . Due to the small frequency offset, the TD single differential scheme outperforms the other schemes at medium and high SNR values. At low SNR, the FD single differential scheme is slightly better than the TD T/2T double differential scheme but at high SNR the latter considerably outperforms the former because the effect of the channel frequency selectivity on the former is more severe than the enlarged noise effect on the latter at high SNR.
Figures 8 and 9 compare the BER performances of the TD single and double (T/2T) differential schemes and the FD single differential scheme at different frequency offsets at $\mathrm{SNR}=10 \mathrm{~dB}$ and $25 \mathrm{~dB}$, respectively. The TD single differential scheme outperforms the other differential schemes at low frequency offsets, and degrades significantly and is outperformed by the other schemes as frequency offset increases. This can be attributed to the frequency-offset-dependent coherence loss in the TD single differential scheme due to phase offset $e^{j 2 \pi f_{0}(N+v) / N}$ which is not encountered in the other two schemes. At small frequency offsets, the larger coherence loss of the FD scheme due to the channel frequency selectivity, and the enhanced noise effect of the TD double differential scheme yield the advantage of the TD single differential scheme. As frequency offset increases, the coherence loss of the TD single differential scheme becomes larger, resulting in worse BER performance than the other two schemes.

The BER performance of TD double differential scheme is better than FD single differential at high SNR and vice versa at low SNR, as can be observed from Figures 8 and 9. The FD single differential scheme suffers from the channel's high frequency selectivity while the TD double differential scheme is affected by the enhanced noise due to the additional differential operation. Furthermore, both time and frequency domain differential schemes are affected by ICI but the natures of ICI terms are different in the two schemes. The decision variable contains correlated ICI terms in the FD scheme but uncorrelated ICI terms in the TD double differential (T/2T) scheme. For the UWB environment, the coherence loss due to the channel frequency selectivity is more dominant than the ICI effect, and hence the TD T/2T differential scheme provides better performance than the FD single differential scheme at high SNR.

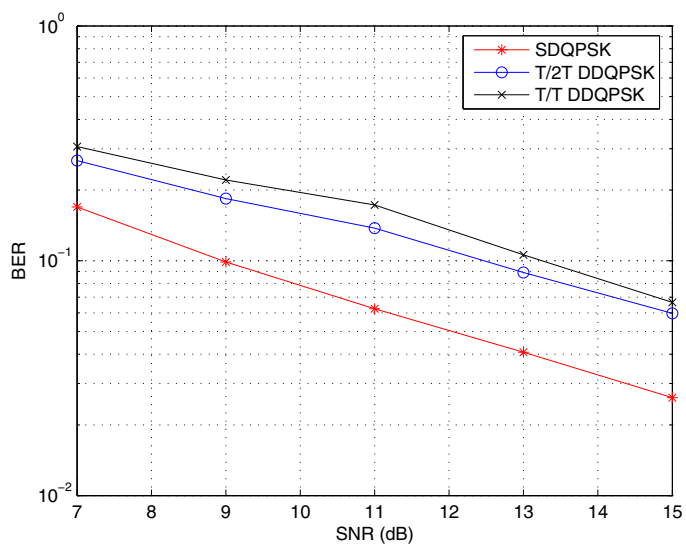

Fig. 5. BER vs. SNR for frequency-domain single and double differential scheme at a normalized frequency offset of 0.07

\section{CONCLUSION}

This paper compares the performance of time and frequency-domain single and double differential OFDM 


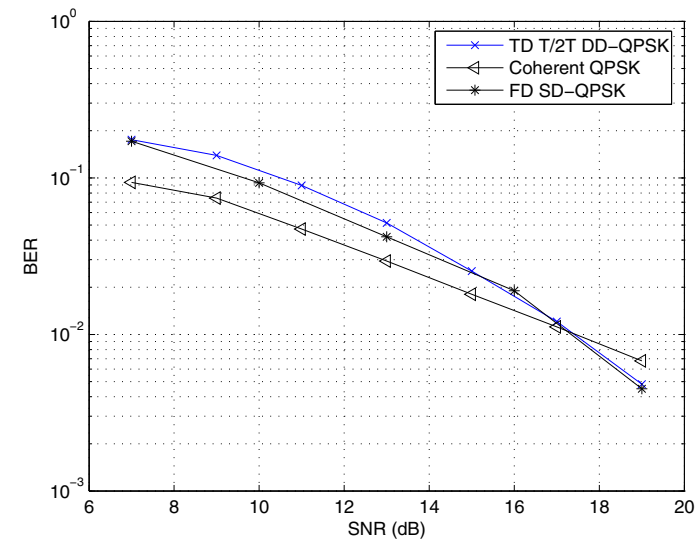

Fig. 6. BER vs. SNR for coherent, time-domain double differential and frequency-domain single differential schemes at a normalized frequency offset of 0.07

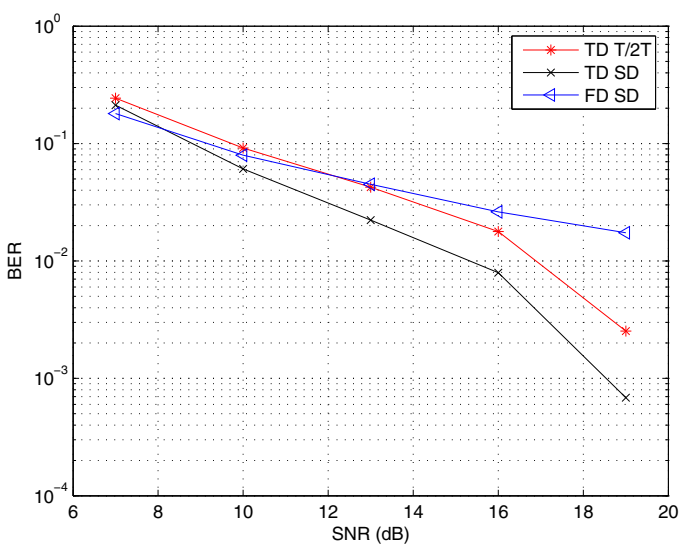

Fig. 7. BER vs. SNR for time-domain single and double differential schemes and frequency-domain single differential scheme at a normalized frequency offset of 0.03

UWB receivers in the presence of frequency offset. We observe that implementing the double differential scheme is helpful only in the time domain. The TD single differential scheme is quite sensitive to frequency offset while the FD single differential scheme is quite robust to frequency offset, especially at low SNR. At low frequency offsets, the TD single differential scheme outperforms the other schemes, while at relatively large frequency offsets the TD double-differential (T/2T) scheme and the FD single differential scheme outperform the TD single differential scheme. At high SNR, the TD double differential $(\mathrm{T} / 2 \mathrm{~T})$ scheme outperforms the FD single differential scheme and the coherent scheme in the presence of frequency offset. At low SNR, the FD single differential scheme outperforms TD double-differential scheme.

\section{REFERENCES}

[1] G. Giannakis and L. Yang, "Ultra-Wideband Communications: An Idea whose Time has Come," IEEE Signal Processing Magazine., vol. 21 , no. 6 , Nov. 2004, pp. 26-54.

[2] A. Batra, J. Balakrishnan, R. Aiello, J. Foerster and A. Dabak, "Design of a Multiband OFDM System for Realistic UWB Channel Environments," IEEE Trans. Microwave Theory and Techniques., vol. 52, no. 9, Part 1, Sep. 2004, pp. 2123-2138.

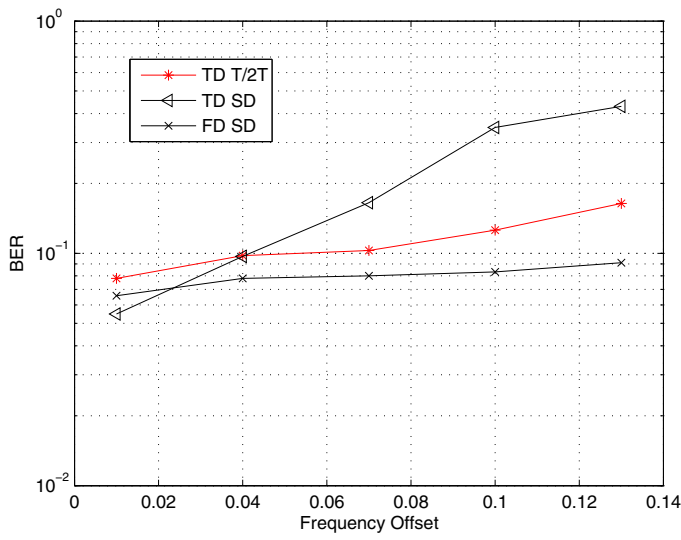

Fig. 8. BER vs. normalized frequency offset for time-domain single and double, and frequency-domain single differential schemes at SNR $=10 \mathrm{~dB}$

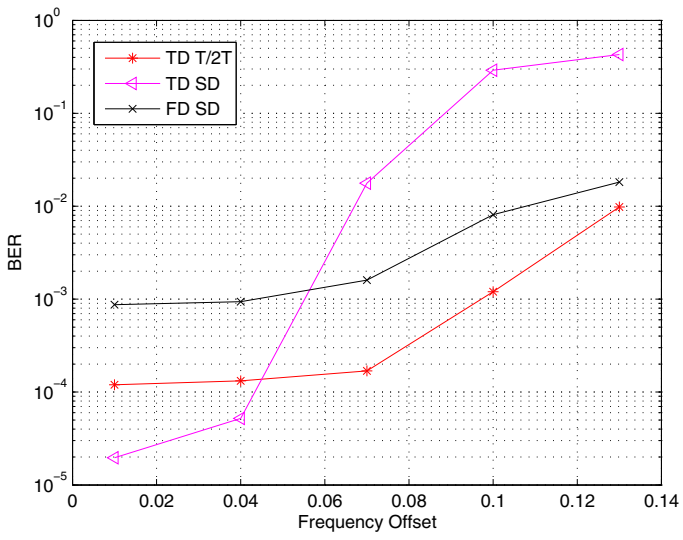

Fig. 9. BER vs. normalized frequency offset for time-domain single and double, and frequency-domain single differential schemes at SNR $=25 \mathrm{~dB}$

[3] D. Divsalar and M. Simon, "Multiple-Symbol Differential Detection of MPSK," IEEE Trans. Commun., vol 38, no. 3, Mar. 1990, pp. 300-308.

[4] M. Ho, S. Somayazulu, J. Foerster and S. Roy, "A Differential Detector for an Ultra-wideband Communications System," IEEE Vehicular Technology Conf., vol. 4, May 2002, pp. 1896-1900.

[5] A. D'Amico and L. Taponecco, "A Differential Receiver for UWB Systems," IEEE Trans. Wireless Commun., vol. 5, no. 7, Jul. 2006, pp. 1601-1605.

[6] J. Lee, D. Toumpakaris, H Lou, and J. Cioffi, "Effect of Carrier Frequency Offset on Time-Domain Differential Demodulation in OFDM Systems," IEEE Vehicular Technology Conf., vol. 1, Sep. 2004, pp. 568-572.

[7] J. Lee, H Lou, D. Toumpakaris, and J. Cioffi, "Effect of Carrier Frequency Offset on OFDM Systems for Multipath Fading Channels," IEEE Global Telecommunications Conf., Vol. 6, Nov.-Dec. 2004, pp. $3721-3725$.

[8] M. Lott, "Comparison of Frequency and Time Domain Differential Modulation in an OFDM System for Wireless ATM," IEEE Vehicular Technology Conf., vol. 2, May 1999, pp. 877-883.

[9] J. Hurst and I. Wassell, "Double Differentially Demodulated Scheme for Short Burst OFDM Systems Operating in Frequency Selective Environments," IEEE Electronics Letters, vol 36, no. 18, Aug. 2000, pp. 1559-1560.

[10] M. Simon and D. Divsalar, "On the Implementation and Performance of Single and Double Differential Detection Schemes,' IEEE Trans. Commun., vol. 40, no. 2, Feb. 1992, pp. 278-291.

[11] P. Stoica, J. Liu and J. Li, "Maximum-Likelihood Double Differential Detection Clarified," IEEE Trans. Information Theory, vol. 50, no. 3, Mar. 2004, pp. 572-576. 